\title{
Increasing the Dynamic Range of a Digitizing Impedance Analyzer Circuit for Resonating Sensors
}

Alexander O. Niedermayer, Thomas Voglhuber-Brunnmaier, Johannes Sell, Bernhard Jakoby

Institute for Microelectronics and Microsensors

Johannes Kepler University

Altenbergerstr. 69, 4040 Linz, Austria

Phone: +43732 24686250

\begin{abstract}
Resonant sensors are used in a wide range of applications, e.g. as microbalances, chemical sensors in liquid and gaseous environments, and for physical property sensing of liquid and viscoelastic media. The signals obtained from these sensors are most often afflicted with unwanted or spurious components which, depending on sensor type and operating conditions, can be caused by parasitic sensor effects or by the implemented sensor interface circuit. Common ways of handling these effects by post-processing the sensor data usually reduce the dynamic range of the measurement interface due to the fact that a certain percentage of the input range is occupied by the unwanted signal components. In this contribution, a method of analog compensation for parasitic signal components is presented, which is implemented in a largely digital impedance analyzer circuit for resonating sensors.
\end{abstract}

\section{Introduction}

An impedance analyzer circuit for resonating sensors [1] was primarily dedicated to evaluate the impedance spectra of quartz crystal resonators (QCR) used for viscosity and density measurements of liquids in a zeolite synthesis experiment which is planned to be conducted aboard the International Space Station (ISS). An analog compensation for spurious signal components caused by parasitic effects in the sensor was introduced and implemented in the analyzer circuit [2]. The implemented signal conditioning concept significantly increases the effective measurement resolution when evaluating strongly damped oscillations of resonating sensors (e.g., QCR operating on higher harmonics in highly viscous media). This is of particular importance for investigations of complex fluids like suspensions, where it is advantageous to perform measurements at multiple frequencies, e.g., by excitation of one resonator at various harmonic vibrations [3]. In this contribution, we present our latest work in this respect, enhancing the compensation concept yielding a optimization boundary for resonant sensor interfaces.

\section{Motivation}

The resonance behavior of thickness shear mode quartz crystal resonators is commonly described by a ButterworthVan Dyke equivalent circuit consisting of the electrode capacitance $C_{0}$ in parallel to a series resonant circuit representing the so called motional branch with its parameters depending on the liquid properties and mass loading $[4,5]$. Harmonic oscillations of the resonator can be modeled by adding corresponding series resonant circuits (Fig. 1).

Each mechanical resonance frequency $\omega_{N}=2 \pi f_{N}$, where $N=(1,3,5, \ldots)$ is the harmonic number of the resonance, is represented by a separate series resonant circuit $\left(C_{N}, L_{N}, R_{N}\right)$.

The motional branch resistance

$$
R_{N}=R_{N 1}+R_{N 2}
$$

comprises the loss resistance of the unloaded resonator $R_{N 1}$ and the resistance $R_{N 2}$ representing the viscous dissipation due to the liquid loading. These two contributions show different dependencies on the harmonic order of the resonance $[5,6]$ yielding $^{1}$

$$
R_{N}=R_{11} \cdot N^{2}+R_{12} \cdot \sqrt{N}
$$

\footnotetext{
${ }^{1}$ Note that Eqn. 2 in [2] the proportionality of $R_{N 2}$ is erroneously denoted as linear instead of square root.
} 


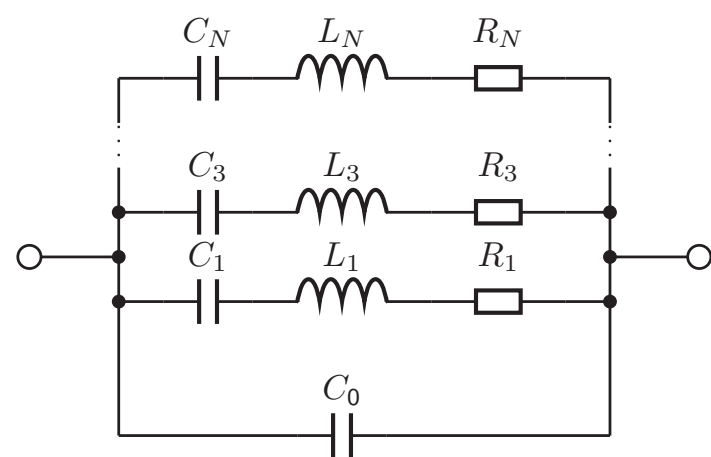

Figure 1: Equivalent electrical network of a QCR for excitation at higher harmonics.

\begin{tabular}{l|ccc|c}
$f_{1}$ & $1.84 \mathrm{MHz}$ & $4.00 \mathrm{MHz}$ & $6.00 \mathrm{MHz}$ & \\
$C_{0}$ & $4.6 \mathrm{pF}$ & $4.7 \mathrm{pF}$ & $4.7 \mathrm{pF}$ & \\
\hline$R_{1}$ & $363 \Omega$ & $23,9 \Omega$ & $19.6 \Omega$ & \\
$R_{3}$ & $10.9 \mathrm{k} \Omega$ & $256 \Omega$ & $796 \Omega$ & \\
$R_{5}$ & $28.8 \mathrm{k} \Omega$ & $1.34 \mathrm{k} \Omega$ & $3.21 \mathrm{k} \Omega$ & air \\
$R_{7}$ & $36.2 \mathrm{k} \Omega$ & & $10.9 \mathrm{k} \Omega$ & \\
\hline$R_{1}$ & $18.6 \mathrm{k} \Omega$ & $8.1 \mathrm{k} \Omega$ & $1.26 \mathrm{k} \Omega$ & \\
$R_{3}$ & $53.2 \mathrm{k} \Omega$ & $22.0 \mathrm{k} \Omega$ & $6.5 \mathrm{k} \Omega$ & IPA \\
$R_{5}$ & $125.5 \mathrm{k} \Omega$ & & &
\end{tabular}

Table 1: Motional branch resistances of some commercial AT-cut QCR for various harmonic resonances when exposed to air or isopropanol (IPA). The values were acquired using an Agilent 4294A impedance analyzer.

Additional damping of resonances is induced by the clamping and mode shape of the resonator which can exceed the above contributions significantly. As example for realistic values the motional branch resistances for different resonators are given in Table 1.

The locus plot of the admittance of a series resonant circuit resembles a circle with a diameter of $R_{N}{ }^{-1}$ and it's center on the real axis. The circle intersects the real axis at the resonant frequency and at the plot origin.

Assuming that the bandwidth $\Delta \omega$ of the resonator is much smaller than a particular resonance frequency $\omega_{N}$ $\left(\Delta \omega<<\omega_{N}\right)$, the admittance circle formed by the series resonant circuit is approximately shifted along the imaginary axis by $j \omega_{N} C_{0}$ due to the current induced by the parallel capacitance as shown in Fig. 2.

For excitation at higher harmonics of the resonator the motional branch resistance as well as the influence of the parallel capacitance $C_{0}$ increases and hence resonant circles become smaller and the dislocation from the plot origin rises (see Fig. 3).

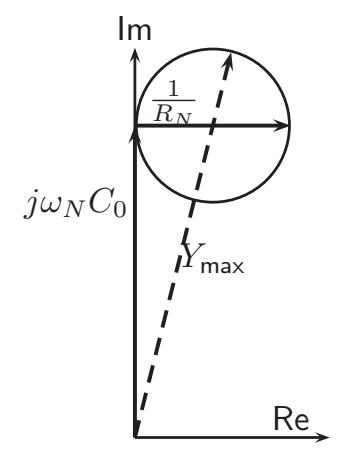

Figure 2: Determination of the maximum amplitude of the sensor admittance.

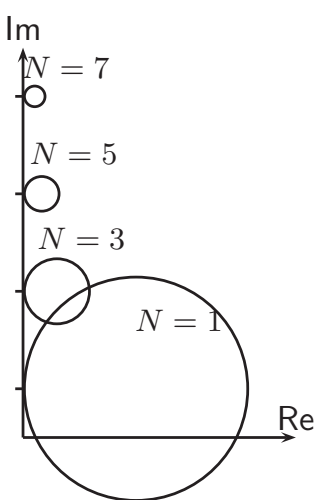

Figure 3: Resonator admittance (simplified) for various harmonic orders $N$.

\section{Dynamic Range of the Sensor Signal}

Series resonant circuits show low impedance when they are in resonance and hence, with respect to the utilization of measurement range, measuring admittance $(Y)$ is preferable to impedance measurement. Consequently, the input swing of the ADC has to cover the maximum occurring sensor admittance. Assuming that the bandwidth $\Delta \omega$ of the resonator is much smaller than a particular resonance frequency $\omega_{N}\left(\Delta \omega<<\omega_{N}\right)$ the maximum of the admittance can be found as shown in Fig. 2 yielding

$$
Y_{\max }=\max |Y|=\left|j \omega C_{0}+\frac{1}{2 R_{N}}\right|+\frac{1}{2 R_{N}}
$$

and with $\omega \approx \omega_{1} \cdot N=\omega_{N}$

$$
Y_{\max } \approx \frac{1}{2 R_{N}}\left(\sqrt{\left(\omega_{1} N\right)^{2} C_{0}^{2} 4 R_{N}^{2}+1}+1\right)
$$


The signal component induced by the parallel capacitance $C_{0}$ is an unwanted parasitic signal (like an offset signal) for the measurement of the motional branch resonance and occupies a certain percentage of the input range of the sensor interface.

As parasitic-free dynamic range (PFDR) of the sensor admittance signal we define the ratio of motional admittance $R_{N}{ }^{-1}$ (i.e. the diameter of the resonance circle) to the maximum occurring sensor admittance $Y_{\max }$ representing a figure of merit for the effectively utilizable resolution of the implemented sensor interface.

When the resonator is significantly damped (e.g. by exposure to a viscous liquid) the influence of the dissipation in the QCR material can be neglected $\left(R_{11}<<R_{12}\right)$ and hence a limit for the PFDR can be given by

$$
\operatorname{PFDR}=\frac{\frac{1}{R_{N}}}{Y_{\max }}<\frac{\frac{1}{R_{12} \sqrt{N}}}{\omega_{1} N C_{0}} \propto N^{-\frac{3}{2}} .
$$

\section{Analog Signal Conditioning}

To maximize the effectively utilizable resolution of digitizing measurement systems, parasitic unwanted signal components have to be suppressed before the signal is sampled by the ADC, similar to the commonly implemented parallel capacitance compensation in analog evaluation circuits $[3,7,8]$. The circuit concept of the implemented compensation is based on a digital synthesizer (Analog Devices AD9959) which is used for generation of the excitation signal for the sensor [1]. An additional channel of the same synthesizer is used to generate a compensation signal which is subtracted from the original sensor signal (Fig. 4). The resulting difference of these two signals is amplified and sampled into the digital domain using a subsampling approach [1]. The additional synthesizer channel provides tunable phase and amplitude and hence can be used to generate any compensation signal of sinusoidal shape.

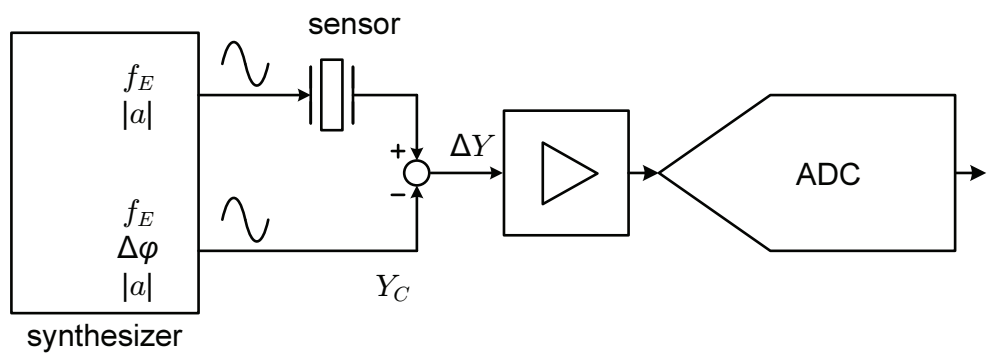

Figure 4: Working principle of the implemented analog compensation. Both synthesizer channels are tunable in amplitude $|a|$ and generate a sinusoidal signal at the excitation frequency $f_{E}$. The compensation channel is additionally tunable in phase $\Delta \varphi$ and can therefor be used to compensate any spurious component in the sensor signal. The difference of both signals is amplified and sampled by the ADC and passed to a signal processor.

\section{Sensor Signal Decomposition}

In this constellation the residual admittance signal $\Delta Y$, which is effectively measured by the ADC, comprises the admittance of the motional branch $Y_{m}$ (which is the desired signal component), the parasitic signal component caused by the parallel capacitance $C_{0}$ and the synthesized compensation signal $Y_{C}$

$$
\Delta Y=Y_{m}+j \omega C_{0}-Y_{C}
$$

By choosing

$$
Y_{C}=j \omega C_{0}
$$

the compensation signal $Y_{C}$ can be used to minimize the parasitic signal component by estimating $C_{0}$ from an uncompensated measurement cycle (where $Y_{C}=0$ ).

With this compensation the PFDR relevant to the ADC is redefined as

$$
\operatorname{PFDR}=\frac{\frac{1}{R_{N}}}{\max |\Delta Y|} .
$$


For repetitive measurements the estimation of $C_{0}$ (and $Y_{C}$ consequently) can be adjusted iteratively if the frequency spectrum is recorded in the vicinity of the resonance. The remaining difference signal $\Delta Y$ can then be amplified to make use of the whole ADC input span [2]. With this approach one can reach PFDR=1 under ideal conditions (with respect to synthesizer resolution and amplification).

However, the PFDR can be further increased by enhancing the compensation signal

$$
Y_{C}^{\prime}=j \omega C_{0}+\frac{1}{2 R_{N}}
$$

with an additional real signal component. In this case, the center of the resonance circle (with the diameter $\frac{1}{R_{N}}$ ) of the remaining signal

$$
\Delta Y^{\prime}=Y_{m}+j \omega C_{0}-Y_{C}^{\prime}
$$

is shifted to the origin of the locus plot, as shown in Fig. 5.

Considering that the ADC input limit also forms a circle in the locus diagram (with its center in the plot origin), one can see that both circles are concentric. This yields the possibility of additionally increasing the analog amplification by a factor of two (Fig. 5).

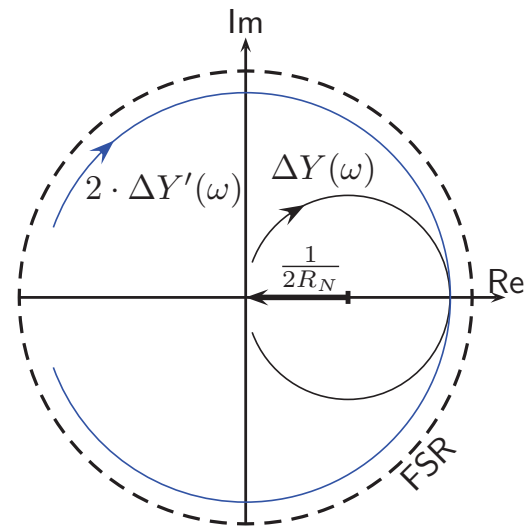

Figure 5: Maximizing the input signal with respect to ADC full scale range (FSR, dashed line) by subtracting $\left(2 R_{N}\right)^{-1}$ from the motional branch admittance $Y_{m}$ and additional amplification before sampling. This approach yields maximum input level over the whole resonance.

For this approach the motional branch resistance $R_{N}$ has to be estimated and tuned in the same way as it is done with $C_{0}$. Iterative estimation of parameters like $C_{0}$ and $R_{N}$ is particularly suitable for the readout of sensors in process monitoring tasks because many repetitions with the same sensor are acquired.

Concluding, the optimization boundary for the PFDR is

$$
\text { PFDR }<2 .
$$

\section{Relation with Auto Balancing Bridge Concept}

The presented compensation method and the circuit concept are related to the auto balancing bridge (ABB) technology as used in laboratory impedance measurement equipment (e.g., [9]). The main difference is that there is no control loop nulling the residual signal at the summing node; instead the residual signal is only reduced and hence the concept represents a kind of "partially balanced interface".

By adapting the circuit for tracking the resonance circle with the compensation signal, the concept would be a partly digital version of the analog control loop in the ABB concept.

\section{Results}

The presented compensation concept was implemented in a single board impedance analyzer circuit based on a synchronous subsampling approach [1].

Sample measurements with the implemented compensation method were acquired using a commercial QCR with a fundamental frequency of $1.8432 \mathrm{MHz}$. The sensor was exposed to isopropanol and the shown resonance characteristics were acquired at the third harmonic of the oscillator. In Fig. 6 the locus plot of the sensor admittance is given with and without application of the described compensation method. As can be seen from 
the amplified signal, the estimation of the circle center of the uncompensated sensor admittance was not perfect in this first iteration. In the figures 7 and 8 the corresponding Bode plots are depicted. Note that the effective parallel capacitance in this experiment is influenced by the permittivity of IPA causing an additional reduction of the PFDR.

The resonances were acquired by sampling the admittance at 101 frequency steps which are non-uniformly distributed according to a Gaussian distribution centered at the resonance frequency. The acquisition took 2.9 seconds per resonance spectrum.

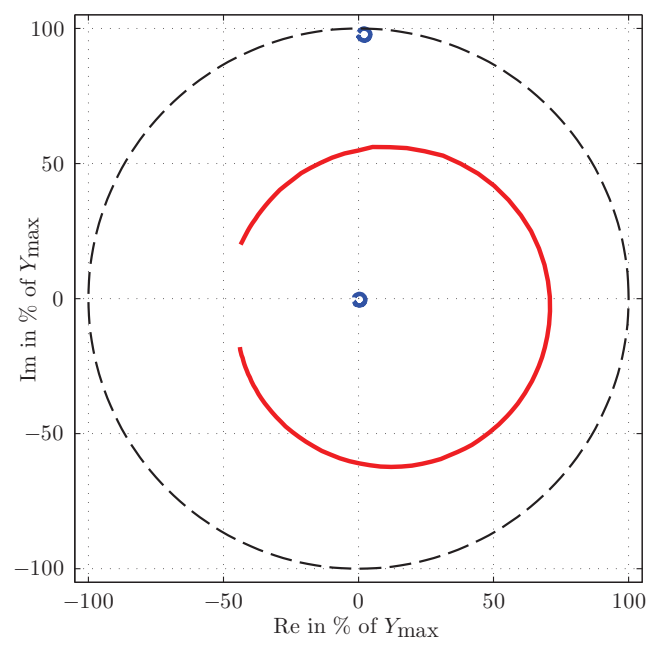

Figure 6: Locus plot of the sensor admittance with and without applying the compensation method. By determining the circle center of the uncompensated sensor resonance (small circle in the top of the figure) and applying an appropriate compensation signal, the resonance circle is shifted to the plot origin, where an additional amplification (in this case $33.6 \mathrm{~dB}$ ) can be applied.

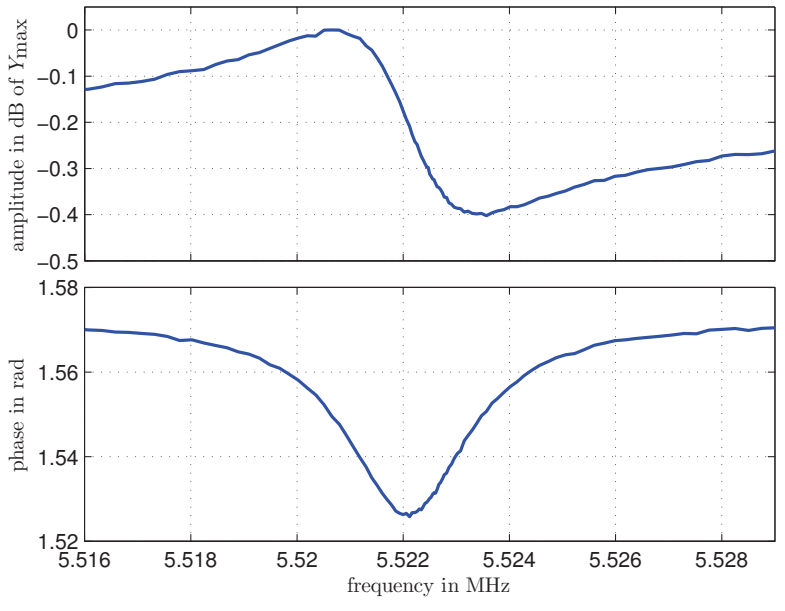

Figure 7: Bode diagram of the uncompensated sensor signal (small circle in the top of Fig. 6). The amplitude was normalized to the maximum occurring admittance $Y_{\text {max }}$.

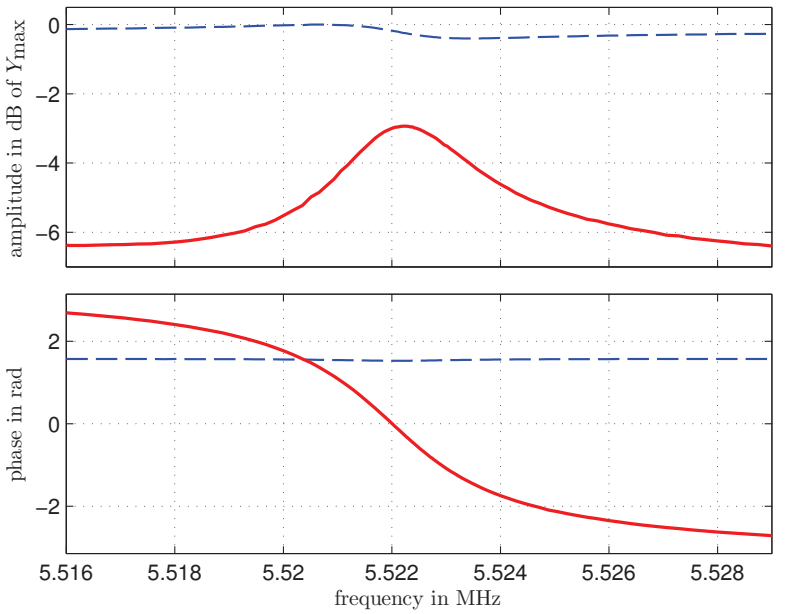

Figure 8: Bode diagram of the compensated sensor signal $\Delta Y^{\prime}$ after amplification. The dashed line shows the original sensor signal (Fig. 7) for comparison. Again the amplitude is given in $\mathrm{dB}$ of $Y_{\max }$.

\section{Conclusions}

Unwanted signal components induced by parasitic sensor effects or sensor interfaces can reduce the effective amplitude resolution of sensor interfaces drastically. Compensation of these components can be advantageous and hence increase the achievable measurement accuracy significantly. For optimal effective resolution, the 
maximum occurring admittance of the resonator can be twice the input span of the interface circuit. Besides QCR, other resonating sensors such as vibrating plates or beam structures with electromagnetic excitation, can be operated with this analyzer circuit.

\section{Acknowledgments}

This work was partially supported by the Austrian Center for Competence in Mechatronics (ACCM) and the Austrian PRIZE program.

\section{References}

[1] A. O. Niedermayer, E. K. Reichel, B. Jakoby, Yet another precision impedance analyzer (yapia) — readout electronics for resonating sensors, Sensors and Actuators A: Physical 156 (2009) 245-250.

[2] A. O. Niedermayer, J. K. Sell, B. Jakoby, Analog compensation of parasitic sensor signals in a subsampling impedance analyzer circuit for resonating sensors, in: Proceedings of the Eurosensors XXIV, Procedia Engineering, Vol. 5, 2010, pp. 57-61.

[3] M. Ferrari, V. Ferrari, K. K. Kanazawa, Dual-harmonic oscillator for quartz crystal resonator sensors, Sensors and Actuators A: Physical 145-146 (2008) 131-138.

[4] B. A. Auld, Acoustic Fields and Waves in Solids, Vol. 1, John Wiley \& Sons, Inc., 1973.

[5] S. Martin, G. Frye, K. Wessendorf, Sensing liquid properties with thickness-shear mode resonators, Sensors and Actuators A: Physical 44 (3) (1994) 209 - 218. doi:10.1016/0924-4247(94)00806-X.

[6] S. J. Martin, V. E. Granstaff, G. C. Frye, Characterization of a quartz crystal microbalance with simultaneous mass and liquid loading, Anal. Chem. 63 (1991) 2272-2281.

[7] F. Eichelbaum, R. Borngräber, J. Schröder, R. Lucklum, P. Hauptmann, Interface circuits for quartz-crystalmicrobalance sensors, Review of Scientific Instruments 70 (5) (1999) 2537-2545. doi:10.1063/1.1149788.

[8] C. Riesch, B. Jakoby, Novel readout electronics for thickness shear-mode liquid sensors compensating for spurious conductivity and capacitances 7 (3) (2007) 464-469. doi:10.1109/JSEN.2007.891931.

[9] Product note 4294a - new technologies for accurate impedance measurement, Tech. rep., Agilent Technologies (2008). 